\title{
LAS ENFERMEDADES INTERCURRENTES DURANTE LA GESTACIÓN Y SUS CONSECUENCIAS SOBRE EL PESO DEL RECIÉN NACIDO
}

\author{
Intercurrencies during pregnancy and their \\ consequences on the weight of the newborn \\ As intercorrências durante a gestação e sulas \\ consequiências sobre o peso do recém-nascido
}

Marli Terezinha Stein-Backes, MSc. *, Maria Cristina Flores-Soares, M.D.**

Recibido: agosto 21/07 - Aceptado: mayo 6/08

\section{RESUMEN}

Objetivo: verificar si las enfermedades intercurrentes presentes en la gestación aumentan las probabilidades de que la madre tenga un niño con bajo peso al nacer (BPN).

Metodología: se trata de un estudio de casos y controles realizado en el periodo de abril a noviembre del 2003, en la ciudad de Río Grande/RS, Brasil. La recolección de datos se hizo a través de un cuestionario aplicado a 547 madres que presentaron parto en las maternidades de los dos hospitales de este municipio.

Resultados: la presencia de hipertensión arterial transitoria o crónica durante la gestación y la amenaza de aborto en el embarazo actual incrementaron el riesgo de las madres de tener un recién nacido de BPN (OR = 3,77; IC 95\% 1,40-10,17 y OR = 6,27; IC 95\% 1,53-25,76, respectivamente).

* Enfermeira, Mestre em Enfermagem/ Hospital-Escola - Universidade Federal de Pelotas (UFPel). Endereço: Rua Gonçalves Chaves 65/Apt 304 "A" - CEP: 96015-560 / Pelotas/RS. Fone: OXX (53)3227-1589. Brasil. E-mail:marli.backes@bol.com.br

** Doutora em Fisiologia da Reprodução / Departamento de Ciências Fisiológicas e Programa de Pós-Graduação em Enfermagem da FURG. Brasil.
Conclusión: la asistencia prenatal diferenciada para las gestantes con hipertensión arterial transitoria y crónica y aquellas que presentaron amenaza de aborto podría ser importante para reducir el bajo peso al nacer asociado.

Palabras clave: bajo peso al nacer, factores de riesgo, obstetricia, amenaza de aborto, epidemiología, técnicas de investigación.

\section{SUMMARY}

Objective: ascertaining whether intercurrences present during pregnancy increased the chances of a mother bearing a child having low birth weight (LBW).

Method: this was a case and control study carried out between April and November 2003 in Rio Grande county/RS, Brazil. Data was collected by means of a questionnaire applied to 547 mothers who gave birth in this county's two hospitals' maternity units.

Outcome: the presence of transitory or chronic arterial hypertension and the threat of miscarriage during pregnancy were positively connected to 
the results $(\mathrm{p}=0.008$ and $\mathrm{p}=0.01$, respectively) thereby increasing the mothers' risk of having LBW babies $(\mathrm{OR}=3.77$; 1.40-10.17 95\% CI and $\mathrm{OR}=6.27 ; 1.53-25.7695 \% \mathrm{CI}$, respectively).

Conclusion: providing differential prenatal assistance to high- and low-risk pregnant women is one of the preventative measures which can be taken for intercurrences during pregnancy and consequently for LBW.

Key words: low birth weight, risk factor, obstetrics, threatened abortion, epidemiology, investigative techniques.

\section{RESUMO}

Objetivo: verificar se as intercorrências presentes na gestação aumentam as chances da mãe ter uma criança com baixo peso ao nascer (BPN).

Metodologia: trata-se de um estudo de casos e controles, realizado no período de abril a novembro de 2003, no município de Rio Grande/RS. A coleta de dados ocorreu através de um questionário aplicado a 547 mães que deram à luz nas maternidades dos dois hospitais deste município.

Resultados: a presença de hipertensão arterial transitória ou crônica durante a gestação e a ameaça de aborto durante a gravidez atual associaram-se positivamente com o desfecho estudado $(\mathrm{p}=0,008$ e p $=0,01$, respectivamente), aumentando o risco da mãe ter um recém-nascido de $\mathrm{BPN}(\mathrm{RO}=3,77$; IC95\% 1,40-10,17 e RO = 6,27; IC95\% 1,53-25,76, respectivamente).

Conclusões: conclui-se que uma das medidas de prevenção das intercorrências durante a gestação e, conseqüentemente, do BPN, é prestar uma assistência pré-natal diferenciada para as gestantes de risco e de alto risco.

Palavras chave: baixo peso ao nascer, fatores de risco, obstetrícia, ameaça de aborto, epidemiologia, técnicas de pesquisa.

\section{INTRODUÇÃO}

A presença de intercorrências na gestação, tais como as patologias maternas, podem afetar a saúde do binômio mãe-filho, não somente pela sua ocorrência, mas também através do efeito originado pelo seu tratamento, inclusive, podem afetar o crescimento fetal e, conseqüentemente, influenciar o peso ao nascer, motivo pelo qual devem ser conhecidas, tratadas e prevenidas. A hipertensão gestacional, quando não tratada, pode desencadear pré-eclâmpsia, eclâmpsia e morte fetal e é também a principal causa de morte materna no mundo inteiro, podendo ainda estar associada à prematuridade, ao retardo do crescimento intrauterino e à mortalidade perinatal. Por outro lado, a infecção do trato urinário na gestação pode originar problemas como a pielonefrite, insuficiência renal e desencadear doenças hipertensivas agudas da gravidez, agravar a hipertensão preexistente e também favorecer o surgimento da anemia na gestante ${ }^{1}$.

O risco de ocorrência de intercorrências durante a gestação varia com o nível socioeconômico, a idade materna, a paridade, a etnia, o peso materno prégestacional, o ganho de peso durante a gestação, e também, com os fatores comportamentais maternos, como o hábito de fumar e ingerir bebida alcoólica. ${ }^{2}$

A fim de detectar as condições patológicas durante a gestação, é necessário ter disponível um programa apropriado de cuidados prénatais, oferecido para a população com suficiente quantidade e qualidade. ${ }^{3}$

Os objetivos almejados com este acompanhamento são o diagnóstico e tratamento de patologias préexistentes e capazes de perturbar o ciclo gravídicopuerperal, a prevenção, diagnóstico e tratamento de patologias próprias da gravidez, a identificação da gestação de alto risco, a orientação higiênicodietética à gestante e o preparo psicofísico para a maternidade. ${ }^{4}$

Os distúrbios hipertensivos durante a gravidez, na maioria das vezes, são graves e acarretam um aumento na incidência do parto cesáreo, sendo que por este motivo exigem intervenção rápida e segura. ${ }^{5}$ Estudo realizado num hospital público de Goiânia, 
no período de janeiro de 1998 a dezembro de 2002, verificou a incidência de recém-nascidos de BPN em mães com doença hipertensiva específica da gravidez e constatou que a mesma está relacionada com a incidência do nascimento de crianças com baixo peso, observando que a idade gestacional não interferiu no resultado. ${ }^{5}$

Atualmente vem ocorrendo uma alta incidência de recém-nascidos com BPN, não só no Brasil como também em outros países e, com tendência de aumentar ainda mais. Dados do Ministério da Saúde ${ }^{6}$ estimam que, dentre todas as gestações, a freqüência de recém-nascidos de BPN é de 10 a 15\%.

Considerando que há vários fatores de risco envolvidos no BPN, desenvolvemos uma pesquisa para avaliar o peso ao nascer em recém-nascidos de mães residentes nas proximidades da área industrial do município de Rio Grande/RS, consideradas mais expostas à poluição ambiental. ${ }^{7}$ Com o intuito de conhecer melhor alguns fatores de risco relacionados com as intercorrências durante a gestação que podem contribuir para esse agravo, realizamos este estudo para verificar se a hipertensão arterial (transitória ou crônica), a diabete (transitória ou crônica), a anemia, a infecção urinária e a ameaça de aborto, presentes na gestação, aumentam as chances da mãe ter uma criança com BPN, uma vez em que ainda há poucos estudos específicos disponíveis sobre o assunto.

\section{METODOLOGIA}

O presente estudo de casos e controles foi realizado no município de Rio Grande/RS, no período de abril de 2003 a novembro de 2003. A coleta de dados ocorreu através de um questionário aplicado, ainda na maternidade, a 547 mães (138 do grupo dos casos, que tiveram recém-nascidos com BPN, ou seja, com peso menor que 2.500 gramas e, 409 do grupo controle, que tiveram recém-nascidos com peso igual ou maior que 2.500 gramas) que deram à luz nos dois hospitais existentes neste município. Buscou-se todos os recém-nascidos de BPN, de partos únicos, neste período (casos). Para cada caso foram incluídos três controles. Os controles foram as três crianças, também de partos únicos que nasceram imediatamente após o caso, no mesmo hospital, também filhos de mães residentes no município de Rio Grande. O cálculo do tamanho da amostra foi definido para alcançar uma precisão de 5\%, com um nível de confiança de 95\%, um poder de $80 \%$ e um risco relativo de 2,50, estando a prevalência de exposição entre os controles em torno de 9\%. Estimou-se que, trabalhando com uma proporção de três controles para cada caso, e incluindo 10\% para perdas e 15\% para controle das variáveis de confusão seriam suficientes 139 casos e 417 controles. Sendo a taxa de prevalência de BPN em Rio Grande em torno de 9,4\%, segundo levantamento realizado para definir a amostragem no qual foi considerado o número total de crianças nascidas no município em outubro de 2002 e, ocorrendo cerca de 3.768 nascimentos ao ano (IBGE, 2003), estimou-se que seriam necessários seis a sete meses para recrutar um número suficiente de casos e controles. Houve um total de três recusas entre os casos e uma recusa entre os controles. Entre os controles houve também quatro perdas.

\section{Variáveis a medir}

Características socioeconômicas e demográficas maternas (renda familiar, presença do companheiro, escolaridade materna, escolaridade paterna, idade materna e cor materna), condições de moradia (tipo de casa, água encanada dentro de casa e tipo de sanitário), características biológicas maternas (peso materno pré-gestacional, altura materna e índice de massa corporal da mãe), fatores reprodutivos (número de gestações anteriores, intervalo interpartal, abortos prévios, natimortos prévios, BPN prévios e pré-termos prévios), fatores ambientais (local de residência materna, tempo de residência materna, local da residência materna anterior e local de trabalho materno), fatores comportamentais (fumo materno, número de cigarros fumados por dia pela mãe, companheiro fumante, número de cigarros fumados por dia pelo 
companheiro, outros fumantes em casa, exposição ao fumo no ambiente de trabalho, consumo de bebida alcoólica pela mãe durante a gestação e trabalho materno fora de casa), ganho de peso, intercorrências na gestação (hipertensão arterial, diabete, anemia, infecção urinária e ameaça de aborto), número de consultas pré-natais e o tipo de parto.

Os dados foram avaliados através do método de regressão logística não condicional, obtendo-se as razões de Odds e intervalos de confiança em todas as análises, utilizando um nível de significância de 0,05. Na análise bivariada, o efeito de cada uma das intercorrências sobre o baixo peso ao nascer foi analisado isoladamente. $\mathrm{Na}$ regressão logística multivariada foi efetuado o ajuste para as variáveis que permaneceram no modelo hierárquico previamente estabelecido, ou seja, que apresentaram um nível de significância menor que 0,20 , que foram a presença do companheiro, escolaridade paterna, idade materna, altura e índice de massa corporal da mãe, intervalo interpartal, natimortos prévios, baixo peso ao nascer prévios, local de residência e local de trabalho da mãe, as quais foram ajustadas com as variáveis do quinto nível do modelo de análise, que foi composto pelas variáveis: fumo materno, número de cigarros fumados por dia pela mãe, companheiro fumante, número de cigarros fumados por dia pelo companheiro, outros fumantes em casa, exposição ao fumo no ambiente de trabalho, consumo de bebida alcoólica pela mãe, trabalho remunerado, ganho de peso, hipertensão arterial, diabete, anemia, infecção urinária, ameaça de aborto, número de consultas pré-natais e tipo de parto.

Quanto às maternidades dos dois hospitais gerais mencinados acima, ambas atendem mulheres de baixa renda, e também, atendem convênios e/ou particulares. Uma delas pertence a um Hospital Universitário (HU) que é referência no atendimento à AIDS, no atendimento à gravidez de alto risco e neonatologia, e também é reconhecido como Hospital Amigo da Criança, desde o ano de 2002. A outra maternidade pertence a uma instituição filantrópica, onde 70\% de seus clientes são atendidos pelo SUS e não possui UTI pediátrica ou neonatologica. Desta forma, a maior parte da amostra deste estudo, ou seja, 60\% das mães eram provenientes do HU.

Os dados referentes ao recém-nascido, bem como os referentes à mãe (nome, peso ao nascer, peso no dia do parto, entre outros), foram levantados a partir dos registros colhidos na maternidade de cada hospital. Além disso, um questionário foi aplicado às mães ainda durante a internação hospitalar na maternidade, por um entrevistador treinado. Com este questionário foram investigados os fatores de risco que poderiam interferir no desfecho estudado. Também foi realizada a mensuração da altura da mãe no final da aplicação do questionário.

Em relação aos aspectos éticos, foi levado em conta o Código de Ética dos Profissionais de Enfermagem e também a Resolução no 196/96 do Ministério da Saúde, sobre pesquisas envolvendo seres humanos.

\section{RESULTADOS}

A presente pesquisa epidemiológica identificou em relação às características socioeconômicas e demográficas maternas, que de todas as variáveis analisadas na análise bivariada, somente a idade materna mostrou associação positiva com o BPN $(\mathrm{p}=0,008)$ Quando se efetuou a análise multivariada, a idade materna mostra tendência a associar-se positivamente com o BPN ( $p=0,052)$, sendo observado um risco maior deste desfecho entre as mães com 35 anos de idade ou mais ( $\mathrm{p}=$ 0,052 e OR $=2,35$ ).

Quanto às características biológicas maternas, na análise bivariada a altura materna apresenta-se como efeito protetor, ou seja, quanto maior a altura da mãe, menor o risco da criança nascer com BPN, $(p=0,002)$. O mesmo ocorre com o peso materno pré-gestacional, ou seja, quando este for maior ou igual a $60 \mathrm{~kg}$, menor o risco $(\mathrm{p}=0,002)$. Já na análise multivariada, a altura materna mantém seu efeito protetor $(\mathrm{p}=0,006)$, e quando a mesma 
era de $160 \mathrm{~cm}$ ou mais, o risco foi ainda menor da criança nascer com BPN ( $\mathrm{RO}=0,43)$, enquanto que o peso materno pré-gestacional não permaneceu no modelo de análise. O índice de massa corporal, embora tendo apresentado tendência de associação negativa em relação ao BPN na análise bivariada (p $=0,062)$, na análise multivariada, o mesmo não foi significativo $(\mathrm{p}=0,11)$.

Já entre os fatores reprodutivos maternos, na análise bruta, a história de natimortos prévios ( $\mathrm{p}$ $=0,03)$ e de BPN prévios $(\mathrm{p}=0,000)$ associaramse positivamente com o BPN, da mesma forma na análise ajustada, $(\mathrm{p}=0,04$ e $\mathrm{p}=0,000$, respectivamente). $\mathrm{O}$ intervalo interpartal não foi significativo neste estudo, nem na análise bivariada $(\mathrm{p}=0,6)$ e nem na multivariada $(\mathrm{p}=0,17)$.

Quanto aos fatores ambientais, em relação ao local de residência materna durante a gestação, há a possibilidade da existência de uma relação entre exposição ambiental a poluentes e BPN no município do Rio Grande/RS, embora não tenha sido encontrada uma associação significativa, $\mathrm{p}=0,067$ na análise bivariada e $\mathrm{p}=0,057$ na análise multivariada. O local de trabalho não apresentou associação com o BPN na análise bivariada ( $\mathrm{p}=$ $0,6)$ e nem na análise multivariada, $(p=0,08)$.

Em relação aos fatores comportamentais, o fumo materno não foi significativo. $\mathrm{O}$ número de cigarros fumados por dia pela mãe encontrou associação positiva com o BPN na análise bivariada $(\mathrm{p}=0,04)$. $\mathrm{Na}$ análise multivariada essa variável não permaneceu no modelo de análise. Já na exposição da mãe ao fumo passivo (do companheiro), ou seja, ao número de cigarros fumados por ele por dia, encontrou-se associação positiva com o BPN na análise bivariada $(\mathrm{p}=$ 0,03). e, na análise multivariada, esta variável apresentou apenas tendência de associação positiva com o desfecho do estudo ( $p=0,09)$. O trabalho materno fora de casa não foi significativo neste estudo. No entanto, as mães que trabalhavam fora de casa representam uma minoria. O consumo de bebida alcoólica pela mãe durante a gestação encontrou apenas tendência de associação positiva com o BPN ( $\mathrm{p}=0,07)$ na análise multivariada.

O ganho de peso materno durante a gestação representa fator de proteção para o BPN, na análise bivariada $(\mathrm{p}=0,00)$ e também na multivariada $(\mathrm{p}$ $=0,0003)$. Da mesma forma o aumento do número de consutas pré-natais (maior que cinco), são fatores de proteção para o BPN ( $\mathrm{p}=0,00$ na análise bivariada e, $\mathrm{p}=0,049$ na análise multivariada).

O tipo de parto não se associou com o BPN na análise bivariada $(\mathrm{p}=0,5)$ e, na análise multivariada o mesmo não permaneceu no modelo de análise.As variáveis sobre as intercorrências na gestação entre os casos e controles e o efeito bruto dessas variáveis sobre o BPN são apresentadas na tabela 1 . A presença de diabete e a ameaça de aborto durante a gestação associaram-se significativamente com o BPN. A hipertensão arterial, anemia e a infecção urinária não foram significativas para o desfecho estudado.

A hipertensão arterial, a diabete, a anemia, a infecção urinária e a ameaça de aborto, foram ajustadas para as variáveis significativas que entraram no modelo de análise $(\mathrm{p}<0,20)$. A partir desse ajuste, as variáveis sobre as intercorrências na gestação que apresentaram-se significativas foram: a ocorrência de hipertensão arterial durante a gestação e a ameaça de aborto durante a gravidez atual. A diabete presente na gestação, apenas apresentou tendência a associar-se positivamente com o BPN. Tabela 2

\section{DISCUSSÃO}

A ocorrência de hipertensão arterial durante a gestação apresentou associação positiva com o BPN. Estudo realizado em Pelotas também encontrou associação positiva entre o BPN e a história de hipertensão arterial. ${ }^{2}$ Há uma maior ocorrência de hipertensão arterial em mães negras durante a gestação, ${ }^{8}$ fato este não foi observado no presente estudo, assim como não houve associação com a idade materna. No entanto, a hipertensão arterial associou-se significativamente com o peso materno pré-gestacional ( $\mathrm{p}=0,007)$ e com o ganho de peso 


\begin{tabular}{|c|c|c|c|c|c|c|}
\hline Variável & \multicolumn{2}{|c|}{ Casos } & \multicolumn{2}{|c|}{ Controles } & Razão de odds (IC 95\%) & $\mathbf{P}$ \\
\hline Hipertensão arterial & \multicolumn{2}{|c|}{$\mathrm{n}=138$} & \multicolumn{2}{|c|}{$\mathrm{n}=407$} & & \\
\hline Não & 102 & $(73,9 \%)$ & 325 & $(79,9 \%)$ & 1,00 & 0,1 \\
\hline Sim & 36 & $(26,1 \%)$ & 82 & $(20,1 \%)$ & $1,39(0,89-2,19)$ & \\
\hline Diabete & \multicolumn{2}{|c|}{$\mathrm{n}=135$} & \multicolumn{2}{|c|}{$\mathrm{n}=404$} & & \\
\hline Não & 122 & $(90,4 \%)$ & 387 & $(95,8 \%)$ & 1,00 & 0,02 \\
\hline Sim & 13 & $(9,6 \%)$ & 17 & $(4,2 \%)$ & $2,42(1,14-5,13)$ & \\
\hline Anemia & \multicolumn{2}{|c|}{$\mathrm{n}=135$} & \multicolumn{2}{|c|}{$\mathrm{n}=401$} & & \\
\hline Não & 82 & $(60,7 \%)$ & 253 & $(63,1 \%)$ & 1,00 & 0,6 \\
\hline Sim & 53 & $(39,3 \%)$ & 148 & $(36,9 \%)$ & $1,10(0,74-1,64)$ & \\
\hline Infecção urinária & \multicolumn{2}{|c|}{$\mathrm{n}=135$} & \multicolumn{2}{|c|}{$\mathrm{n}=409$} & & \\
\hline Não & 78 & $(57,8 \%)$ & 236 & $(57,7 \%)$ & 1,00 & 0,9 \\
\hline Sim & 57 & $(42,2 \%)$ & 173 & $(42,3 \%)$ & $0,99(0,67-1,47)$ & \\
\hline Ameaça de aborto & \multicolumn{2}{|c|}{$\mathrm{n}=138$} & \multicolumn{2}{|c|}{$\mathrm{n}=409$} & & \\
\hline Não & 125 & $(90,6 \%)$ & 392 & $(95,8 \%)$ & 1,00 & 0,02 \\
\hline Sim & 13 & $(9,4 \%)$ & 17 & $(4,2 \%)$ & $2,39(1,13-5,07)$ & \\
\hline
\end{tabular}

Fonte: BACKES, 2004. ${ }^{7}$

Tabela 2. Distribuição entre casos e controles do efeito bruto das variáveis sobre as intercorrências na gestação associadas com o BPN, ajustadas para as características socioeconômicas e demográficas maternas, características biológicas maternas, fatores reprodutivos maternos, fatores ambientais,

fatores comportamentais durante a gestação, ganho de peso, número de consultas pré-natais e tipo de parto significativos.

Variável Casos

Controles

Razão de odds Bruta (IC 95\%)

\section{Razão de odds $\quad$ P ajustada (IC 95\%)}

\begin{tabular}{|c|c|c|c|c|c|c|c|}
\hline Hipertensão arteriala & \multicolumn{2}{|c|}{$\mathrm{n}=138$} & \multicolumn{2}{|c|}{$\mathrm{n}=407$} & & & \\
\hline não & 102 & $(73,9 \%)$ & 325 & $(79,9 \%)$ & 1,00 & 1,00 & 0,008 \\
\hline $\operatorname{sim}$ & 36 & $(26,1 \%)$ & 82 & $(20,1 \%)$ & $1,39(0,89-2,19)$ & $3,77(1,40-10,17)$ & \\
\hline Diabetea & \multicolumn{2}{|c|}{$\mathrm{n}=135$} & \multicolumn{2}{|c|}{$\mathrm{n}=404$} & & & \\
\hline não & 122 & $(90,4 \%)$ & 387 & $(95,8 \%)$ & 1,00 & 1,00 & 0,08 \\
\hline $\operatorname{sim}$ & 13 & $(9,6 \%)$ & 17 & $(4,2 \%)$ & $2,42(1,14-5,13)$ & $4,65(0,82-26,40)$ & \\
\hline Ameaça de aborto & \multicolumn{2}{|c|}{$\mathrm{n}=138$} & \multicolumn{2}{|c|}{$\mathrm{n}=409$} & & & \\
\hline não & 125 & $(90,6 \%)$ & 392 & $(95,8 \%)$ & 1,00 & 1,00 & 0,01 \\
\hline $\operatorname{sim}$ & 13 & $(9,4 \%)$ & 17 & $(4,2 \%)$ & $2,39(1,13-5,07)$ & $6,27(1,53-25,76)$ & \\
\hline
\end{tabular}

Fonte: BACKES, 2004.

a = variáveis que permaneceram no modelo de análise, ajustados para presença do companheiro, escolaridade paterna, idade materna, altura, IMC, intervalo interpartal, natimortos prévios, BPN prévios, local de residência e local de trabalho maternos. 
durante a gestação $(p=0,003)$. Mães com peso pré-gestacional maior ou igual a 60 quilos tiveram mais chance de desenvolver hipertensão arterial durante a gravidez (57\%) quando comparadas com as mães com peso pré-gestacional inferior a 60 quilos (42,9\%). Da mesma forma o ganho de peso. Uma maior proporção de mães que aumentaram mais de 12 quilos na gravidez, apresentou hipertensão arterial $(55,5 \%)$ do que as mães que aumentaram até 12 quilos (44,6\%). Opostamente, o fumo materno durante a gestação apresentou tendência a associar-se negativamente com a hipertensão arterial. Mães que fumaram durante a gestação tiveram menos hipertensão arterial $(25,4 \%)$ do que as mães que não fumaram $(74,6 \%)$. No estudo de coorte realizado em seis capitais brasileiras, os distúrbios hipertensivos foram mais freqüentes entre as mulheres obesas. ${ }^{9}$ Alta prevalência de hipertensão transitória e crônica durante a gestação foi encontrada em Pelotas/RS. ${ }^{1}$ A hipertensão arterial transitória ou crônica pode levar ao retardo do crescimento intra-uterino, prematuridade e alta mortalidade perinatal, ${ }^{10} \mathrm{O}$ que poderia explicar a sua associação com o BPN encontrada no presente estudo.

A presença de diabete durante a gestação apenas mostra uma tendência de associação positiva com o BPN ( $p=0,08)$. Associação negativa foi encontrada em Pelotas entre o BPN e a história de diabete. ${ }^{2}$ Estudo de coorte realizado em serviços de pré-natal geral do Sistema Único de Saúde em seis capitais brasileiras entre 1991 e 1995 constatou que as mães que apresentaram um risco aumentado para a diabete gestacional eram obesas a apresentaram menor risco para microssomia. ${ }^{9}$ Embora o esperado para os filhos de mães diabéticas é que os mesmos sejam macrossômicos, este estudo mostra que as mães que apresentaram diabete durante a gestação possuem tendência a terem recém-nascidos com BPN, o que pensamos poderia estar associado à presença de hipertensão arterial, decorrente da obesidade, ou de outros fatores de risco relacionados, como por exemplo, a renda familiar. As mães com renda familiar acima de dois salários mínimos, apresentaram tendência a desenvolver diabete gestacional ( $p=0,07)$.

A ameaça de aborto durante a gravidez associouse positivamente com o BPN nos dois tipos de análise. Embora não se tenha encontrado estudos disponíveis na literatura que associem esta variável com o BPN, sabemos que as mães que apresentam ameaça de aborto durante a gestação, possuem uma probabilidade maior de terem recém-nascidos prematuros e com BPN, devido a uma série de intercorrências que podem acometê-las, tais como, descolamento prematuro da placenta, placenta prévia, bolsa rota, trabalho de parto prematuro, entre outras. Da mesma forma como a diabete, a ameaça de aborto igualmente manifestou-se num pequeno número de mães.

Finalmente, a infecção urinária não apresentou associação com o BPN na análise bruta e nem na análise ajustada. Em Pelotas no entanto, foi encontrada associação positiva entre o BPN e a infecção do trato urinário. ${ }^{2}$

A respeito dos fatores de proteção neste estudo: o aumento da altura ( $\geq 160 \mathrm{~cm}$ ), o ganho de peso na gestação e o número de consultas pré-natais ( $\geq$ 5), Ortiz ${ }^{11}$ aponta dois indicadores responsáveis pelo BPN relacionados com o estado nutricional da mãe durante o período pré-gestacional, ou seja, o peso menor que $50 \mathrm{~kg}$ e a estatura inferior a 1,49 $\mathrm{cm}$ e sugere melhorar as condições nutricionais das mulheres em idade fértil, como uma intervenção adequada para diminuir o BPN.

Os dados deste estudo podem apontar subsídios valiosos para o planejamento de políticas públicas, contribuindo especialmente para a melhoria dos serviços oferecidos às gestantes, pelo fato de proporcionar uma melhor compreensão dos eventos ligados ao BPN.

\section{CONCLUSÕES}

De todas estas intercorrências que podem ocorrer durante a gestação, levantadas neste estudo, apenas a presença de hipertensão arterial transitória ou crônica 
durante a gestação e a ameaça de aborto presente na gravidez associaram-se significativamente com o BPN. Além disso, a presença de diabete transitória ou crônica, também apresentou tendência para tal. Em contrapartida, são fatores de proteção neste estudo: o aumento da altura ( $\geq 160 \mathrm{~cm}$ ), o ganho de peso na gestação e o número de consultas pré-natais $(\geq 5)$.

Tornam-se necessários neste município, outros estudos para avaliar e esclarecer melhor as intercorrências que ocorrem durante a gestação e suas conseqüências sobre o peso do recém-nascido, bem como os efeitos dos fatores de risco sobre o BPN, inclusive através da utilização de outras metodologias.

\section{REFERÊNCIAS}

1. Garcias GL. Diagnóstico comunitário e análise do discurso a respeito da compreensão popular em relação à causalidade dos defeitos congênitos [tese]. Porto Alegre: Programa de Pós-Graduação em Genética e Biologia Molecular, Universidade Federal do Rio Grande do Sul;1999.

2. Santos IS. Consumo de cafeína e baixo peso ao nascer: um estudo de casos e controles de base populacional [tese]. Porto Alegre: Universidade Federal do Rio Grande do Sul - Faculdade de Medicina;1995.

3. De Aquino MM, Cecatti JG, Mariani Neto C. Risk factors associated to fetal death. Sao Paulo Med J 1998;116:1852-7.
4. Kruse W, Abeche AM. Assistência pré-natal. Porto Alegre: Ed. Universidade-UFRGS;1992.

5. Martins CA, Rezende LPR, Vinhas DCS. Gestação de alto risco e baixo peso ao nascer em Goiânia. Rev Eletrônica de Enfermagem [online].2003;5:49-55. Capturado em 2004 Jun 20. Disponível em: http:// www.fen.ufg.br/revista.

6. Brasil. Ministério da Saúde. Secretaria de Políticas de Saúde. Gestação de alto risco. Brasília: Ministério da Saúde;2000.

7. Backes MTS. O baixo peso ao nascer em recémnascidos de mães residentes nas comunidades próximas ao parque industrial do município de Rio Grande(RS): um estudo de casos e controles [dissertação]. Rio Grande: Fundação Universidade Federal do Rio Grande, Programa de Pós-Graduação em Enfermagem;2004.

8. Cecatti JG, Machado MR, dos Santos FF, Marussi EF. Curva dos valores normais de peso fetal estimado por ultra-sonografia, segundo a idade gestacional. Cad Saude Publica 2000;16:1083-90.

9. Nucci LB, Schmidt MI, Duncan BB, Fuchs SC, Fleck ET, Santos Britto MMS. Nutritional status of pregnant women: prevalence and associated pregnancy outcomes. Rev Saude Publica 2001;35:502-7.

10. Cunninghan FG, Lindheimer MD. Hypertension in pregnancy. N Engl J Med 1992;326:927-32.

11. Ortiz EI. Estrategias para la prevención del bajo peso al nacer en una población de alto riesgo, según la medicina basada en la evidencia. Colomb Med 2001;32:159-62. 